\title{
The Application of Intelligent Face Recognition Technology in Commercial Bank System in the Era of Big Data
}

\author{
He Yuanzi \\ Nanchang Institute of Science and Technology, Nanchang, China
}

Keywords: the era of big data; intelligent face recognition technology; commercial bank system; application

\begin{abstract}
With the progress of science and technology, biological recognition technology develops rapidly, so that previous identity recognition is gradually being replaced. However, face recognition technology, as an important part of biological recognition technology, is gradually accepted in identity recognition with its stability, convenience and inclusiveness. Besides, it is widely applied especially in bank system. The effective application of face recognition technology can further optimizes the bank system, improve the security of the system and provide high-quality services. Therefore, this paper discusses the intelligent face recognition technology in the era of big data, analyzes its specific contents and explores its practical application in commercial bank system, so as to give fully play to this technology and promote the rapid development of commercial bank.
\end{abstract}

With the economic development and changes of social trend, China's commercial banks are required to conduct effective reforms on their operation mode. Especially, in recent years, with the advent of big data era, the effective utilization and intellutalized development of big data resources has become an important path for commercial banks to enhance their comprehensive strength. Face information has many advantages, simple and vivid design, which cannot be stolen or replicated. It is the strategic resources for commercial banks to explore and construct under the background of big data. In the development of science and technology, the costs for constructing face database and related recognition system is lower, and the accuracy of recognition is also improving. Therefore, in addition to guaranteeing secure services, in order to further improve the work efficiency, it is necessary to conduce in-depth research on the specific application of face recognition technology.

\section{Introduction to Intelligent Face Recognition Technology}

Face recognition technology, whose main goal is identity retrieval and validation, is the means to extract face information from dynamic or static images and match with face identity collected in database. Compared with other recognition technologies, such as fingerprint recognition, palm print recognition, iris recognition, and identity recognition, the recognition accuracy of face recognition is lower due to light, orientation, occlusion, expression and other factors. However, in terms of acquisition method, this technology has strong friendliness. It does not require the cooperation of the parties, and the information collection and identification can be completed when the parties are not aware of it. Therefore, this technology has become one of key research projects in intelligence field. Over the years of development, it has been gradually mature and already effectively applied in various fields, including access control, security and protection, financial field and so on.

Based on application situation, face recognition can be specifically divided into four types, including three-dimensional face recognition that mainly recognizes depth information acquisition and matching, multi-modal face recognition mainly for sketch, thermal infrared or infrared imaging, face recognition set for surveillance video, and face recognition set for two-dimensional image. According to these face recognition technologies with data input, experts and scholars in related fields put forward face recognition technology methods based on different scientific backgrounds, models and suppositions, which also are similar in processing procedures after conclusion. Specifically, the first is face detection, which can solve problems of where the face is and the number of faces, mainly to detect and determine the position of face in videos or pictures and 
conduct effective separation processing; the second is face tracking, which can solve problems of face movement, to track faces in each frame of video. If there is occlusion in this process, the tracking can be continued after the occlusion. For instance, keep tracking after many interfaced faces can avoid the mistake; the third is face standardization to guarantee the accurate position of the five sense organs, which involves pre-processing, face standardization and normalization; the fourth is face recognition to retrieve faces and determine the identity information of face [1].

When constructing face recognition system and related database, face data shall be modelled and trained. If the database uses dynamic update, it will involve some contents of online learning. In face recognition, it is required to compare the faces to be recognized with face data in database, judge the similarity of the both, and complete retrieval and validation according to preset standards. There are many methods of face recognition, such as related methods based on neural network, model, geometrical characteristic, and template.

At present, the method based on deep learning has higher recognition accuracy and it is widely applied in the field. The deep learning here, is not specific algorithm, but the general item of deep belief networks, RBM, Coding and Sparse. This method based on neural network, combined with cognitive psychology, is the simulation of human brain neural signal transmission, which is different from the original 2-3 layer training layer in the neural network model. Deep learning can guarantee 8-9 layer training layer. Whereas, in the initial stage, due to higher calculation complexity and the great training data volume, previous hardware is too weak. However, with the rapid development of computer software, the accuracy of deep learning algorithm is enhanced further. Besides, Baidu, Microsoft and Google conduct in-depth research on deep learning technology, many face recognition teams based on deep learning appear in the market, and this approach based on deep learning has gradually become an important direction of face recognition technology [2].

Currently, the commonly used standards of face recognition algorithm mainly include FRVT and LFW. The LFW database is constructed by University of Massachusetts at Amherst, including more than 13,000 pictures mainly coming from Internet. However, in database, there are more than one picture for one figure, and sometimes there is only one picture of one figure. But, these pictures are shot in daily environment, so those are full with strong practicality, and the validation results are persuasive. The test has higher efficient of difficulty, whereas, the industry and academic field all participated in it. FRVT is mainly built by American institute of technology and national standards organization, which aims at testing the algorithm performance of face recognition in the industry. The standard test is to perform non-scheduled tests based on the database with 1,600,000 people, which is only open for the industry [3].

\section{The Specific Application of Intelligent Face Recognition Technology in Commercial Bank System}

All the time, face recognition technology is mainly applied in public safety, including criminal tracking, driving license verification, airport security, video surveillance and so on. However, in recent years, it is gradually applied in commercial bank system. In the future, commercial bank can effectively use it from safety monitoring and controlling and business promotion, so as to realize the full implementation of face recognition technology.

\subsection{Safety monitoring and controlling}

In the bank, the difficult problem of safety monitoring and controlling is mainly to monitor multiple moving targets in real time in dynamic scenarios. The face recognition technology can perform multi-target retrieval and comparison in areas with high personnel density like bank, with good application effect. And face information acquisition is easy, not easy to be stolen and copied, with natural and intuitive features. Therefore, face recognition technology can be considered as an important means for safety monitoring and controlling in commercial banks, which is shown respectively. 


\subsubsection{The controlling of personnel influences in business places}

In commercial bank, camouflage detection in face recognition can be applied to monitor business places so as to ensure the safety of bank business activities. It is mainly to recognize personnel with occluded face in business places. The system can carry out real-time comparison with various identity data in police database, and call the police in time through Internet or issue the blacklist early-warning when discovering abnormal conditions. What's more, it can submit the acquired data of criminal suspects to police security organization, so that the case detection and latter early-warning can obtain corresponding data support [4].

\subsubsection{The identity recognition of personnel in commercial vaults}

Due to the higher requirement of security in business places of bank, so the requirement of identity recognition technology is also higher, especially in special environment, such as ATM room, escort vehicle, and vault, so many traditional identity recognition technologies cannot satisfy the requirement of security. For instance, access card can be lost, fingerprint can be copied and verification code can be stolen. Whereas, face recognition technology has the function of vivo detection, can overcome the drawbacks and further improve the quality of safety monitoring and controlling for the bank [5].

\subsubsection{The intelligent recognition alarm in ATM}

The typical application of automatic equipment in bank is ATM machine, which also widely applies face recognition technology. For instance, the camera on ATM machine can recognize the identity of people and compare the bank card registration information, shown in Figure 1, to avoid the credit card fraud. At the same time, it can also identify the people with deliberately occluded face or camouflage and compare with police database, so that the security of people withdrawing can be guaranteed. The system can trigger the preset alarm rule to effectively protect the life and property security of customers. In addition, face recognition system can monitor customers' finance, and inform them in real time to further improve the user experience [6].

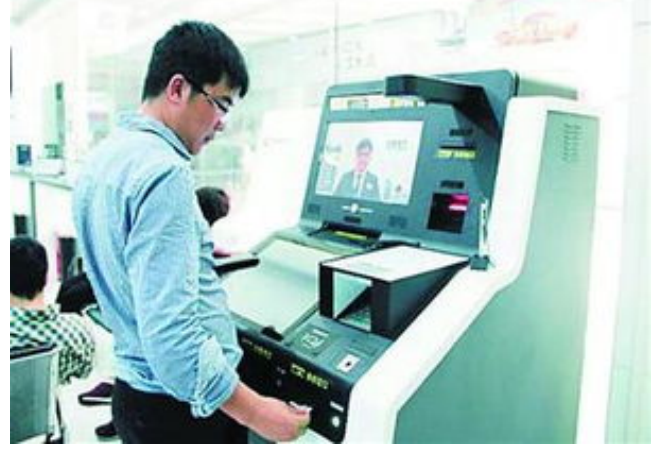

Figure 1

\subsection{Business promotion}

At present, the application of face recognition technology in business promotion is still in initial stage, which requires constant exploration and optimization. Its application methods are presented.

\subsubsection{It can realize remote account opening and logging-in.}

In bank, interview signing is an important part of the account opening operation, which not only takes up customers' time, but also increases the bank's human resources consumption. The application of face recognition technology can replace traditional recognition with naked eyes, which is shown in Figure 2, reduce the investment in time and costs, complete the processing procedures of personal data filling and interview signing of account opening and card obtaining, improve greatly user experience, recognize the customer identity and credit in the entire network and reduce the experience factor and psychological factor. Besides, in remote logging-in through online bank, customers can use face recognition instead of password input and other operations, 
which can smoothly complete the operation of demand transfer, personal card transfer, bill inquiry and credit card repayment, which can effectively reduce the password forgotten or stolen [7].

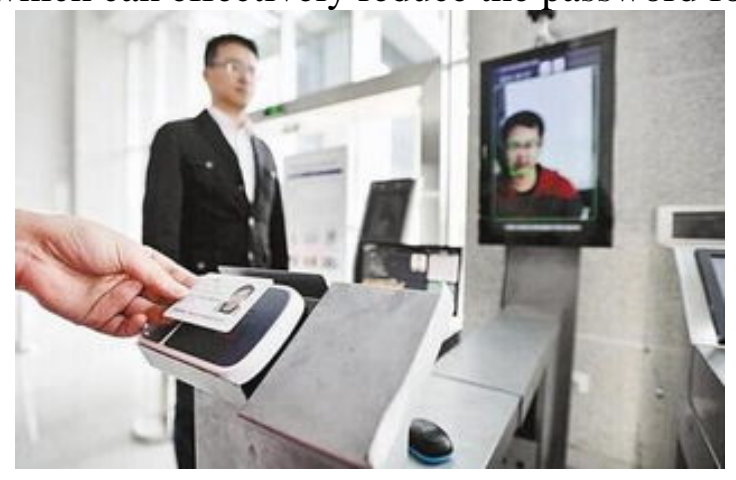

Figure 2

\subsubsection{It can satisfy the personalized demand of customers.}

With the improvement of economic system, the competition faced by commercial banks is also intensifying. The market share has changed from the original product orientation to the current service orientation. The setting of personalized service functions can effectively improve user experience, which has become an important direction in the future development of commercial banks. The effective application of face recognition technology can perform accurate search of customer identity and information. After the customer is included in business network, face recognition technology can quickly determine whether it is a customer of the bank and obtain the customer's basic information like name and age, which is helpful for the staff to understand the customer and provide good service to the customer. In addition, the customer behavior simulation can extract and analyze the customer's previous business habits, transaction flow and product purchase, thereby improving the pertinence of customer product recommendation and effectively improving the marketing success rate [8].

\subsubsection{It can issue the loan.}

When banks issue loans, in order to avoid the malicious fraud or impersonation loans, face recognition technology can be applied for effective prevention and controlling. According to the face information input of the loan customer in the network, using data sharing, it can recognize and verify the identity of the customer within the entire network. Only when the identity information and the bank database information are accurately matched, the loan is formally approved, so that the identity verification of loan customers can achieve network, intelligent and information management [9].

It can be seen that face recognition technology has wide application prospect in commercial bank. Commercial banks, in the application of this technology, shall combine their own business development, start from business promotion, actively implement and apply face recognition in customer services. Specifically, on the one hand, from the perspective of customer, its application in customer services can effectively solve their urgent demand so that they can obtain better user experience, and improve customers' satisfaction; on the other hand, in terms of commercial bank, its application in customer services can efficiently improve bank profit, which is conductive to the comprehensive implementation of face recognition technology [10].

\section{Conclusion}

In short, face recognition technology, widely applied in identity verification, can effectively identify face information so that banks can comprehensively obtain customer information, which can improve service quality. Therefore, in its application, commercial banks shall conduct in-depth research and constantly optimize its application methods, so as to give fully play to the function of this technology and effectively promote the development of commercial bank. 


\section{Acknowledgements}

Fund Project: Key lab project in Nanchang City, Jiangxi Province. Project Name: Key Lab of Intelligent Building Network Project in Nanchang. Project No. 2013ZDSY003

\section{References}

[1] Wang Yanbo, Gao Qian, Yang Xuan. Applications of Intelligent Face Recognition for Commercial Banking in Big Data Era [J]. The Banker, 2016,8(2):104-106.

[2] Zhang Zhongbao. On the Application of Face Recognition in Commercial Bank -Based on Computer Vision [J]. Computer CD Software and Applications, 2013,5(20):61-62.

[3] Che Zhihong, Jiao Zilu, Liu Rong. Research and Application of Face Recognition Technology [J]. Computer Programming Skills \& Maintenance, 2017,3(16):78-81.

[4] Li Qian. Face Recognition Technology and Its Application [J]. Heilongjiang Science and Technology Information, 2014,6(1):60.

[5] IS' Vision. The Application of Human Face Detection and Recognition in Bank [J]. China Auto-ID, 2013,7(2):64-65.

[6] Luo Wenyi. The Design and Achieve of ATM Authentication System Based on Face Recognition Technology [D]. South China Agricultural University, 2016.

[7] Tian Zhijin. Research on Security Technologies of Bank Vault [J]. Telecom World, 2018,4(5):283-284.

[8] Ma Shenglan. Bank Customers Recognition System Based on Face Layered Filter [J]. Computer Systems \& Applications, 2017,9(2):99-103.

[9] Wu Min, Lai Junliang. PKI Teller Identity Certification System Based on Face Recognition [J]. Technology and Market, 2013,5(9):9-11.

[10] Lei Wen, Zhao Pan, Liang Xingjian. Design of System Avoiding Bankcard Being Stolen and Robbed Based on Identity Authentication on Face Geometry [J]. Journal of Chengdu University (Natural Science), 2013,9(3):243-245,249. 